\title{
Neutron stars: Cosmic laboratories for matter under extreme conditions
}

\author{
Ignazio Bombaci ${ }^{1,2}$ and Domenico Logoteta ${ }^{2}$ \\ ${ }^{1}$ Dipartimento di Fisica, Università di Pisa, Pisa, Italy \\ ${ }^{2}$ INFN, Sezione di Pisa, Pisa, Italy
}

\begin{abstract}
The true nature and the internal constitution of the compact stars known as neutron stars (NSs) is one of the most fascinating enigma in modern astrophysics. We discuss some of the present models for the internal structure of NSs and the connection with the properties of ultra dense hadronic matter. In particular, we discuss the role of strangeness on the equation of state and the implications of the measurement of 2 solar mass NSs in PSR J1614-2230 and PSR J0348+0432.
\end{abstract}

Neutron stars (NSs) are incomparable natural laboratories that allow us to investigate the fundamental constituents of matter and their interactions under extreme conditions that cannot be reproduced in terrestrial laboratories. The bulk properties and the internal constitution of NSs primarily depend on the equation of state (EoS) of strong interacting matter [1], i.e. on the thermodynamical relation between the matter pressure, energy density and temperature. Determining the correct EoS model describing NS is a fundamental problem of nuclear physics and astrophysics, and a major effort has been made during the last few decades to solve it by measuring different NS properties using the data collected by various generations of $\mathrm{X}$-ray and $\gamma$-ray satellites and by ground-based radio telescopes. The rather recent accurate measurement of the masses, $M=1.97 \pm 0.04 M_{\text {sun }}[2]$ and $M=2.01 \pm 0.04 M_{\text {sun }}$ [3], of the neutron stars in PSR J1614-2230 and PSR J0348+0432 respectively, has ruled out all the EoS models which cannot support such high values of stellar masses. 
Due to the large values of the stellar central density, various particle species and phases of dense matter are expected in NS interiors. Thus different types of "neutron stars" are hypothesized to exist. In the simplest and conservative picture the core of a NS is modeled as an uncharged uniform fluid of neutrons, protons, electrons and muons in equilibrium with respect to the weak interactions ( $\beta$-stable nuclear matter). These are the so-called nucleon stars. Even in this simplified picture, the microscopic determination of the EoS from the underling nuclear interactions remains a formidable theoretical problem. In fact, one has to determine the EoS to extreme conditions of high density and high neutron-proton asymmetry, i.e. in a regime where the EoS is poorly constrained by nuclear data and experiments. The nuclear symmetry energy is thus one of the most relevant quantities to control the composition, and the pressure of $\beta$-stable nuclear matter [4], and therefore many NS attributes such as the radius, moment of inertia, and crustal properties [5].

Another important issue is related to the role of three-nucleon interactions (TNIs) on the EoS at high density. In fact, it is well known that TNIs are essential to reproduce the experimental binding energy of few-nucleon $(\mathrm{A}=3,4)$ systems and the empirical saturation point $\left(n_{0}=0.16 \mathrm{fm}^{-3}\right.$, $\left.(E / A)_{0}=-16 \mathrm{MeV}\right)$ of symmetric nuclear matter [6]. As shown by various microscopic calculations [7-10] of the EoS of $\beta$-stable nuclear matter based on realistic nucleon-nucleon $(\mathrm{NN})$ interactions supplemented with TNI, it is possible to obtain NS sequences with maximum mass $M_{\max }>2 M_{\text {sun }}$ and thus in agreement with presently measured masses. However, the value of $M_{\max }$ strongly depends on the strength of the TNI at high density and in addition the central densities for the maximum mass configurations are in the range $n_{c}\left(M_{\max }\right)=(6-8) \rho_{0}$.

At these high densities hyperons are expected among the stellar constituents. The reason for hyperons formation is very simple, and it is mainly due to the fermionic nature of nucleons, which makes the neutron and proton chemical potentials very rapidly increasing functions of the density. As soon as the chemical potential $\mu_{n}$ of neutrons becomes sufficiently large, the most energetic neutrons (i.e. those on the Fermi surface) can decay via the weak interactions into $\Lambda$ hyperons and form a new Fermi sea for this hadronic species with $\mu_{\Lambda}=\mu_{n}$. The $\Sigma^{-}$can be produced via the process $e^{-}+n \rightarrow \Sigma^{-}+\nu_{e}$ when the $\Sigma^{-}$chemical potential fulfill the condition $\mu_{\Sigma^{-}}=\mu_{n}+\mu_{e}$ (we consider neutrino-free matter). Other hyperons can be formed with similar weak processes.

To study the influence of hyperons on NS structure, we have performed a Brueckner-Hartree-Fock calculation of the EoS of hyperonic matter using 
the following interactions: the Argonne v18 (Av18) NN interaction [11]; the TNI used in [8] to reproduce the empirical nuclear matter saturation point; the the Nijmegen ESC08b potential [12] to describe the hyperon-nucleon (NY) interaction. No three-body interactions of the type nucleon-nucleonhyperon (NNY) and NYY, YYY have been considered.

The chemical potentials $\mu_{i}$ for the different stellar constituents calculated in this scheme are shown in Fig. 1 (upper panel). The onset of $\Lambda\left(\Sigma^{-}\right)$occurs at $n=0.35 \mathrm{fm}^{-3}\left(0.64 \mathrm{fm}^{-3}\right)$, thus at a density well below the central density $n_{c}^{\max }=1.02 \mathrm{fm}^{-3}$ for the $M_{\max }$ pure nucleonic star calculated within the same approach and the Av18+TNI interaction. The composition of $\beta$-stable hyperonic matter is reported in Fig. 1 (lower panel). Notice that at $n=5 n_{0}$ hyperons represent about $43 \%$ of the total number of baryons. The effect of hyperons on the EoS is shown in Fig. 2 (upper panels), where we compare the EoS for $\beta$-stable pure nucleonic matter (curves Av18+TNI) with that of $\beta$-stable hyperonic matter (curves Av18+TNI+ESC08b). As we can see the presence of hyperons produces a significant reduction of the pressure of the system (upper right panel in Fig. 2). As a consequence, solving the relativistic stellar structure equations, we find an impressive decrease of the stellar maximum mass from $M_{\text {max }}=2.28 \mathrm{M}_{\text {sun }}$ to $M_{\max }=1.38 \mathrm{M}_{\text {sun }}$ when hyerons are included among the stellar constituents. The prediction of a value for $M_{\max }<2 M_{\text {sun }}$ is a common feature of many present calculations

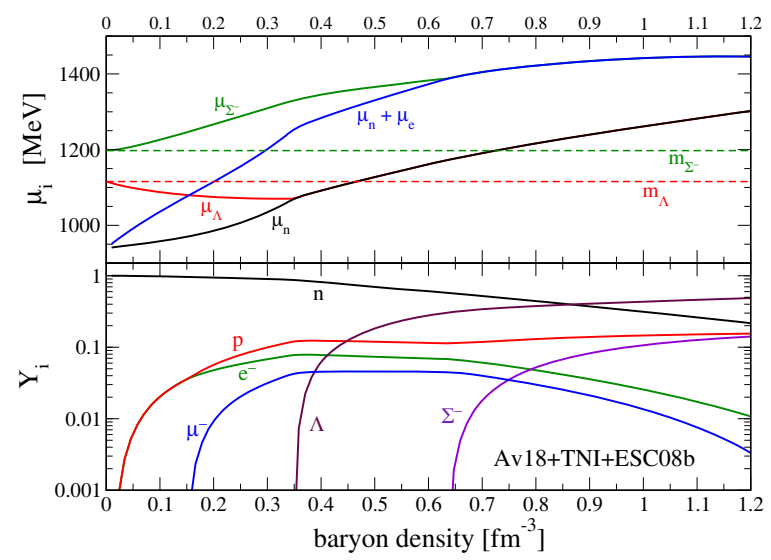

Figure 1: Chemical potentials (upper panel) and concentrations (lower panel) of the different stellar constituents in $\beta$-stable hyperonic matter as a function of the total baryon density. 
[10,13-15] based on microscopic hyperonic matter EoSs.

Thus, on the one hand the presence of hyperons seems unavoidable, on the other hand their presence results in a stellar maximum mass not compatible with measured NS masses. This baffling problem is known as the "hyperon puzzle" in NSs. Clearly, one should try to trace back the origin of this problem to the underlying NY and YY two-body interactions or to the possible repulsive NNY, NYY and YYY three-body interactions. Presently, this is a very active research field. Thus, the use of microscopic EoS of hyperonic matter in the contest of NS physics is of fundamental
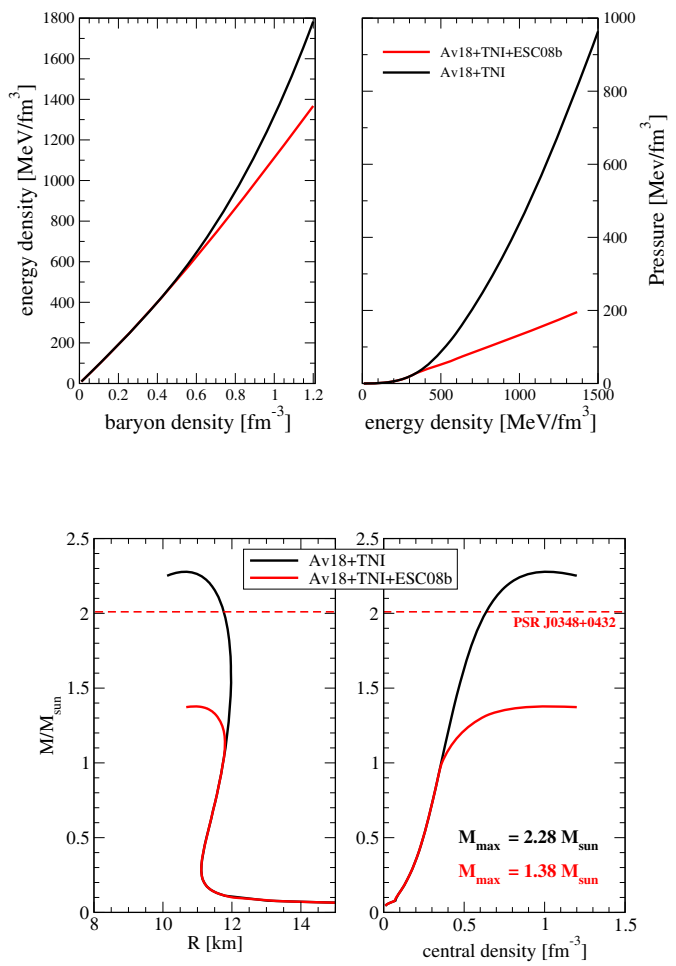

Figure 2: Upper panels: EoS of $\beta$-stable matter as a function of the total baryon density. The upper (lower) curves refer to the case of nuclear (hyperonic) matter. Lower panels: gravitational mass as a function of the stellar radius (lower left panel) and of the central baryon density (lower right panel) in the case of nucleon stars (upper curves) and hyperon stars (lower curves). The dashed horizontal line represents the measured mass $M=2.01 \pm 0.04 M_{\text {sun }}$ [3] of PSR J0348+0432. The mass of the Sun is denoted as $M_{\text {sun }}=1.99 \times 10^{33} \mathrm{~g}$. 
importance for the understanding of strong interactions involving hyperons, and to learn how these interactions behave in a dense many-body system.

The core of a massive NS is one of the best candidates in the Universe where a transition from a phase where quarks are confined within baryons and mesons ("hadronic matter") to a quark deconfined phase ("quark matter") could occur. In the low temperature $T$ and high baryon chemical potential region of the QCD phase diagram (which is the one relevant for NS physics) several QCD inspired models suggest the deconfinement transition to be a first-order phase transition [16]. As it is well known, all first order phase transitions are triggered by the nucleation of a critical size drop of the new (stable) phase in a metastable mother phase. This is a very common phenomenon in nature (e.g. fog or dew formation in supersaturated vapor, ice formation in supercooled water).

One of the most exciting astrophysical consequences of the nucleation process of quark matter (QM) in the core of massive hadronic stars (HSs) (i.e. NSs in which no fraction of QM is present) is that above a threshold value of their mass, HSs are metastable [17-19] to the "decay" (conversion) to quark stars (QSs) (i.e. to hybrid stars or to strange stars). This stellar conversion process liberates a huge amount of energy (a few $10^{53} \mathrm{erg}$ ) and it could be the energy source of Gamma Ray Bursts (GRBs) [20]. In addition, within this scenario, one has two coexisting families of compact stars in the Universe: pure hadronic stars and quark stars $[17,18]$. The members of these two families could have similar values for their gravitational masses but different values for their radii [18].

The metastability of HSs originates from the finite size effects (which represents the driving "force" of first order phase transitions) in the formation process of the first QM drop in the hadronic environment.

In cold $(T=0)$ bulk matter the deconfinement transition takes place at static transition point defined by the Gibbs' criterion for phase equilibrium

$$
\mu_{H}=\mu_{Q} \equiv \mu_{0}, \quad P_{H}\left(\mu_{0}\right)=P_{Q}\left(\mu_{0}\right) \equiv P_{0}
$$

where $\mu_{H}=\left(\varepsilon_{H}+P_{H}\right) / n_{H}$ and $\mu_{Q}=\left(\varepsilon_{Q}+P_{Q}\right) / n_{Q}$ are the Gibbs energies per baryon (average chemical potentials) for the hadron and quark phase respectively, $\varepsilon_{H}\left(\varepsilon_{Q}\right), P_{H}\left(P_{Q}\right)$ and $n_{H}\left(n_{Q}\right)$ denote respectively the total (i.e., including leptonic contributions) energy density, the total pressure and baryon number density for the hadron (quark) phase.

Consider now the more realistic situation in which one takes into account the energy cost due to finite size effects in creating a drop of deconfined QM in the hadronic environment. As a consequence of these effects, the 
formation of a critical-size drop of QM is not immediate and it is necessary to have an overpressure $\Delta P=P-P_{0}$ with respect to the static transition point. Thus, above $P_{0}$, hadronic matter is in a metastable state, and the formation of a real drop of QM occurs via a quantum nucleation mechanism. Thus, a HS having a central pressure larger than $P_{0}$ is metastable with respect to the conversion to a QS. These metastable HSs have a mean-life time which is related to the nucleation time to form the first critical-size drop of deconfined matter in their interior ${ }^{1}$. The critical mass $M_{c r}$ of the metastable HS is defined $[17,18]$ as the value of the gravitational mass for which the nucleation time is equal to one year: $M_{c r} \equiv M_{H S}(\tau=1 \mathrm{yr})$. Pure hadronic stars with $M_{H}>M_{c r}$ are very unlikely to be observed. $M_{c r}$ plays the role of an effective maximum mass for the hadronic branch of compact stars [18]. While the Oppenheimer-Volkov maximum mass $M_{H S, \max }$ is determined by the overall stiffness of the EOS for hadronic matter, the value of $M_{c r}$ will depend in addition on the bulk properties of the EOS for quark matter and on the properties at the interface between the confined and deconfined phases of matter (e.g., the surface tension $\sigma$ ).

These findings are exemplified in Fig. 3, where we show the mass-radius (MR) curve for hadronic stars (HS) and that for quark stars (QS). The configuration marked with an asterisk on the hadronic MR curve represents the HS for which the central pressure is equal to $P_{0}$ and thus the nucleation time $\tau_{q}=\infty$. The full circle on the HS sequence represents the critical mass configuration $M_{c r}$, whereas the full circle on the QS curve represents the QS which is formed from the conversion of the HS with $M^{H S}=M_{c r}$. As we can see, for the EoS parametrizations used in the calculations reported in Fig. 3 (see figure caption for informations on the EoS parametrizations), PSR B1913+16 (which has a mass $M=1.4398 \pm 0.0002 M_{\text {sun }}$ ) can be interpreted as a pure HS, whereas PSR J1614-2230 is more likely a QS.

We assume [20] that during the stellar conversion process the total number of baryons in the star (or in other words the stellar baryonic mass $\left.M_{B}\right)$ is conserved. Thus the total energy liberated in the stellar conversion is given by [20] the difference between the critical mass HS $\left(M_{c r}\right)$ and that of the final QS $\left(M_{f i n}\right)$ configuration with the same baryonic mass: $E_{c o n v}=\left(M_{\text {in }}-M_{f i n}\right) c^{2}$. It has been shown $[17,18,20]$ that $E_{c o n v}=0.5-$ $4.0 \times 10^{53} \mathrm{erg}$. This huge amount of released energy will cause a powerful neutrino burst, likely accompanied by intense gravitational waves emission,

${ }^{1}$ The actual mean-life time of the HS will depend on the mass accretion or on the spin-down rate which modifies the nucleation time via an explicit time dependence of the stellar central pressure. 
and conceivably it could cause a second delayed (with respect to the supernova explosion) explosion. Under favorable physical conditions this second explosion could be the energy source of a powerful GRB [17,20]. Thus this scenario is able to explain a "delayed" connection between supernova explosions and GRBs.

The stellar conversion process, described so far, will start to populate the new branch of quark stars, i.e. the part of the QS sequence above the full circle (see Fig. 3). Long term accretion on the QS can next produce stars with masses up to the maximum mass $M_{\max }^{Q S}$ for the quark star configurations. Thus within this scenario one has two coexisting families of compact stars: HSs and QSs [18]. The quark star branch is occasionally referred to as the "third family" of compact stars, considering white dwarfs as the first family and HSs as the second family. Notice also that there is a range of values of stellar gravitational mass (see Fig. 3) where HSs and QSs with the

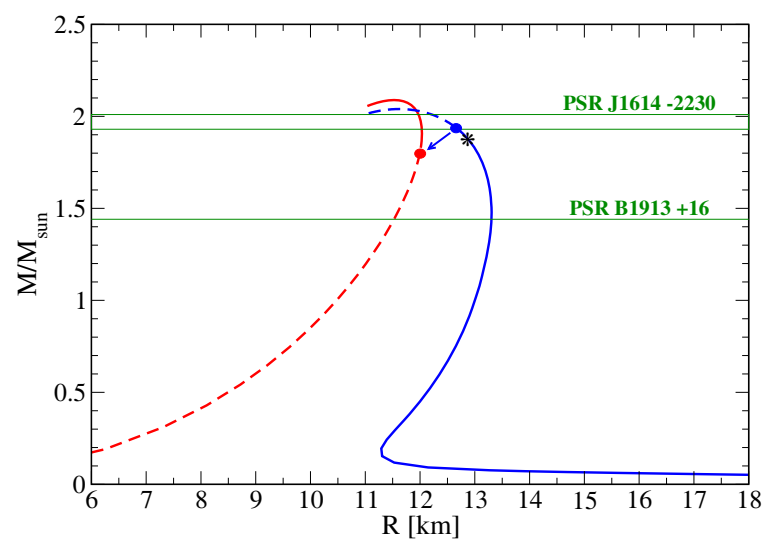

Figure 3: Mass-radius relation for hadronic star (HS) and quark stat configurations (QS). The configuration marked with an asterisk represents the HS for which the nucleation time $\tau_{q}=\infty$ (i.e. $P_{c}=P_{0}$ ). The conversion process of the HS, with a gravitational mass equal to the critical mass $M_{c r}$, into the final QS is denoted by the full circles connected by an arrow. Results are relative to the the GM1 model [21] of hyperonic matter with hyperon- $\sigma$ meson coupling $x_{\sigma}=0.7$, and for strange star configurations with the extended bag model EOS of Ref. [22] with $B_{\text {eff }}=$ 47.2 MeV/ $\mathrm{fm}^{3}$ and $a_{4}=0.7$. The values of the critical gravitational (baryonic) mass and of the final QS mass are calculated for a surface tension $\sigma=10 \mathrm{MeV} / \mathrm{fm}^{2}$. The lower horizontal line represents the mass $M=1.4398 \pm 0.0002 M_{\text {sun }}$ of the pulsar PSR B1913+16, whereas the higher horizontal lines represent the mass $M=$ $1.97 \pm 0.04 M_{\text {sun }}$ of PSR J1614-2230 [2]. 
same gravitational mass can exist ("twin stars").

\section{References}

[1] M. Prakash et al., Phys. Rep. 280 (1997) 1.

[2] P. Demorest, et al., Nature, 467 (2010) 1081.

[3] J. Antoniadis et al., Science, 340 (2013) 1233232.

[4] I. Bombaci and U. Lombardo, Phys. Rev. C 44, (1991) 1892.

[5] J.M. Lattimer and A.W. Steiner, Eur. Phys, J. A 50 (2014) 40.

[6] D. Logoteta, et al., Phys. Rev. C 91 (2015) 064001.

[7] R.B. Wiringa, V. Fiks, and A. Fabrocini, Phys. Rev. C 38 (1988) 1010.

[8] M. Baldo et al., Astron. Astrophys. 328 (1997) 274.

[9] A. Akmal et al., Phys. Rev. C 58, (1998) 1804.

[10] Z.H. Li and H.-J. Schulze, Phys. Rev. C 78 (2008) 028801.

[11] R.B. Wiringa et al., Phys. Rev. C 51, (1995) 38.

[12] Th.A. Rijken et al., Nucl. Phys. A 835 (2010) 160.

[13] M. Baldo et al., Phys. Rev. C 61 (2000) 055801.

[14] I. Vidaña et al., Phys. Rev. C 62 (2000) 035801.

[15] H.-J. Schulze and Th. A. Rijken, Phys. Rev. C 84 (2011) 035801.

[16] S.D.H. Hsu, M. Schwetz, Phys. Lett. B 432 (1998) 203; Z. Fodor, S.D. Katz, JHEP 04 (2004) 050.

[17] Z. Berezhiani et al., Astrophys. Jour. 586 (2003) 1250.

[18] I. Bombaci, I. Parenti, I. Vidaña, Astrophys. Jour. 614 (2004) 314.

[19] A. Drago, A. Lavagno and G. Pagliara, Phys. Rev. D 69 (2004) 057505.

[20] I. Bombaci and B. Datta, Astrophys. Jour. Lett. 530 (2000) L69.

[21] N.K. Glendenning, S.A. Moszkowski, Phys. Rev. Lett. 67 (1991) 2414.

[22] E. Fraga et al., Phys. Rev. D 63 (2001) 121702(R); S. Weissenborn et al., Astrophys. Jour. 740 (2011) L14. 